\title{
Squeezing and Sub-Poissonian Effects of Light in Third Harmonic Generation
}

\author{
R. PrataP ${ }^{a, *}$, D.K. GIRI ${ }^{b}$ AND A. PRASAD ${ }^{c}$ \\ ${ }^{a}$ Department of Physics, R.S. More College, Vinoba Bhave University, Hazaribag, Govindpur, India \\ ${ }^{b}$ Department of Physics, Sindri College, Vinoba Bhave University, Hazaribag, Sindri, India \\ ${ }^{c}$ Department of Physics, P.K.R.M. College, Vinoba Bhave University, Dhanbad, India
}

(Received May 20, 2013; in final form January 9, 2014)

\begin{abstract}
The squeezing and sub-Poissonian effects of light in third harmonic generation are investigated based on the fully quantum mechanical approach up to the first order Hamiltonian interaction in $g t$, where $g$ is the coupling constant between the modes per second and $t$ is the interaction time between the waves during the process in a nonlinear medium. The coupled Heisenberg equations of motion involving real and imaginary parts of the quadrature operators are established. The occurrence of amplitude squeezing effects in both the quadratures of the radiation field in the fundamental mode is investigated and found to be dependent on the selective phase values of the field amplitude. The photon statistics of the pump mode in this process have also been investigated and found to be sub-Poissonian in nature. It is shown that for particular phase values the amplitude squeezing and sub-Poissonian photon statistics of light occur simultaneously. It is observed that there is no possibility to produce squeezed light in the harmonic mode up to first-order interaction in $g t$. Further, it is found that the normal squeezing in the harmonic mode directly depends upon the amplitude-cubed squeezing of the initial pump field to the case of second-order interaction in $g t$. This gives a method of converting higher-order (amplitude-cubed) squeezing of the fundamental mode into normal squeezing of the harmonic mode and vice versa.
\end{abstract}

DOI: $10.12693 /$ APhysPolA.125.1126

PACS: 42.50.Ar, 42.50.Dv, 42.65.Ky

\section{Introduction}

Squeezing and sub-Poissonian photon statistics of the electromagnetic field [1], which is a purely quantum mechanical phenomenon [2] has attracted considerable attention owing to its low-noise property $[3,4]$ with applications in high quality telecommunication [5], quantum cryptography [6] etc. Squeezing has been either experimentally observed or theoretically predicted in a variety of nonlinear optical processes, such as harmonic generation $[7,8]$, multi-wave mixing processes [9-11], Raman [12, 13], hyper-Raman [14] etc. Hong and Mandel [15] and Hillery [16] have introduced the notion of amplitude squeezing of the quantized electromagnetic field in various nonlinear optical processes. Squeezing and photon statistical effect of the field amplitude in harmonic generation has also been reported by Mandel [17]. Higher-order sub-Poissonian photon statistics of light have also been studied by Kim and Yoon [18]. Recently, Prakash and Mishra [19] have reported the higher-order sub-Poissonian photon statistics and their use in detection higher-order squeezing. The nonclassical phenomena squeezing of radiation and photon statistics effects are expected to manifest itself in optical processes in which the nonlinear response of the system to the radiation field plays a great role. It also represents a new type of quantum state of the electromagnetic field and

\footnotetext{
*corresponding author; e-mail: dkgscism@yahoo.co.in, rajendra.pratap.08@gmail.com
}

it has always been of interest to the research community in the fields of quantum optics, nonlinear optics, atomic physics, molecular physics, and biological physics; hence their study can be expected to lead to new fundamental insights.

The aim of this paper is to study further properties of amplitude squeezing and sub-Poissonian effects of the electromagnetic field in the fundamental mode including harmonic mode in third-harmonic generation (THG) process under short-time approximation based on a fully quantum mechanical approach up to first order in $g t$. The paper is organized as follows. Section 2 gives the definitions of squeezing and sub-Poissonian states of light. We establish the analytic expression of selective phase angle dependent amplitude squeezing and sub-Poissonian light in the fundamental mode up to first-order in $g t$ in Sect. 3. The photon statistics of the pump mode in this process have also been incorporated in this section and found to be sub-Poissonian in nature. In Sect. 4, we study the occurrence of amplitude squeezing effects in both the quadrature of the radiation field in the harmonic mode and found to be dependent on amplitude-cubed squeezing of the fundamental mode. Finally, we conclude the paper in Sect. 5 .

\section{Definitions of amplitude squeezing and sub-Poissonian states of light}

Squeezed states of light are characterized by reduced quantum fluctuations in one quadrature of the field at the expense of the increased fluctuations in the other quadrature. It is possible to characterize the amplitude by its 
real and imaginary parts as

$$
X_{1}=\frac{1}{2}\left(A+A^{\dagger}\right) \quad \text { and } \quad X_{2}=\frac{1}{2 \mathrm{i}}\left(A-A^{\dagger}\right),
$$

where $A$ and $A^{\dagger}$ are the slowly varying operators useful in discussing squeezing effects. For a single mode of the electromagnetic field with frequency $\omega$ and creation (annihilation) operators $a^{\dagger}(a)$, they are given by

$$
A=a \exp (\mathrm{i} \omega t), \quad A^{\dagger}=a^{\dagger} \exp (-\mathrm{i} \omega t) .
$$

The operators defined by Eq. (1) do not commute and obey the commutation relation

$$
\left[X_{1}, X_{2}\right]=\frac{\mathrm{i}}{2}
$$

and, as a result, satisfy the uncertainty relation $(\hbar=1)$ :

$$
\Delta X_{1} \Delta X_{2} \geq \frac{1}{4}
$$

where $\Delta X_{1}$ and $\Delta X_{2}$ are the uncertainties in the quadrature operators $X_{1}$ and $X_{2}$, respectively. A quantum state is squeezed in the $X_{1}$ direction if $\Delta X_{1}<\frac{1}{2}$ and is squeezed in the $X_{2}$ direction if $\Delta X_{2}<\frac{1}{2}$.

Amplitude squared squeezing [16] is defined in terms of operators $Y_{1}$ and $Y_{2}$ as

$$
Y_{1}=\frac{1}{2}\left(A^{2}+A^{\dagger 2}\right) \quad \text { and } \quad Y_{2}=\frac{1}{2 \mathrm{i}}\left(A^{2}-A^{\dagger 2}\right)
$$

These operators obey the commutation relation

$$
\left[Y_{1}, Y_{2}\right]=\mathrm{i}\left(2 N_{A}+1\right),
$$

where $A^{\dagger} A=N_{A}$ is the photon number operator in mode $A$.

The commutation relation (6) leads to the uncertainty relation

$$
\Delta Y_{1} \Delta Y_{2} \geq\left\langle\left(N_{A}+\frac{1}{2}\right)\right\rangle,
$$

where $\Delta Y_{1}$ and $\Delta Y_{2}$ are the uncertainties in the quadrature operators $Y_{1}$ and $Y_{2}$, respectively. A quantum state is squeezed in the $Y_{1}$ direction if $\left(\Delta Y_{1}\right)^{2}<\left\langle\left(N_{A}+\frac{1}{2}\right)\right\rangle$ and is squeezed in the $Y_{2}$ direction if $\left(\Delta Y_{2}\right)^{2}<$ $\left\langle\left(N_{A}+\frac{1}{2}\right)\right\rangle$.

Amplitude-cubed squeezing [16, 22] is defined by the operators

$$
Z_{1}=\frac{1}{2}\left(A^{3}+A^{\dagger 3}\right) \quad \text { and } \quad Z_{2}=\frac{1}{2 \mathrm{i}}\left(A^{3}-A^{\dagger 3}\right) .
$$

The operators obey the commutation relation

$$
\left[Z_{1}, Z_{2}\right]=\frac{\mathrm{i}}{2}\left(9 N_{A}^{2}+9 N_{A}+6\right),
$$

which leads to the uncertainty relation

$$
\Delta Z_{1} \Delta Z_{2} \geq \frac{1}{4}\left\langle\left(9 N_{A}^{2}+9 N_{A}+6\right)\right\rangle .
$$

Amplitude-cubed squeezing is said to exist if

$$
\left(\Delta Z_{1}\right)^{2} \quad \text { or } \quad\left(\Delta Z_{2}\right)^{2}<\frac{1}{4}\left\langle\left(9 N_{A}^{2}+9 N_{A}+6\right)\right\rangle .
$$

The quantum effect of sub-Poissonian photon statistics is the reduction of quantum fluctuations in photon number and is reflected by an increase of fluctuations of phase of the field. Hence the photon number uncertainty [17] is

$$
\left\langle\left(\Delta N_{A}\right)^{2}\right\rangle<\left\langle N_{A}\right\rangle \text {. }
$$

\section{Squeezing and sub-Poissonian effects of light in the fundamental mode}

THG is a process in which an incident laser beam of the fundamental frequency $\omega_{1}$ interacts with a nonlinear medium to produce the harmonic frequency at $\omega_{2}=3 \omega_{1}$. This model is chosen to make the model realistic and our discussions hold for all similar models.

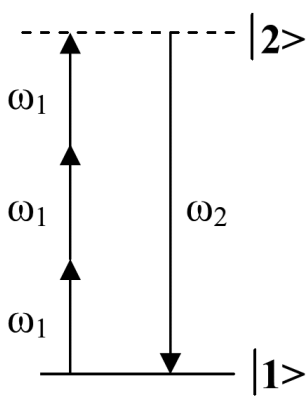

Fig. 1. Third harmonic generation model.

In this model (Fig. 1), the interaction is looked upon as a process involving absorption of three pump photons of frequency $\omega_{1}$ each and system going from $|1\rangle$ to $|2\rangle$ and emission of one photon of frequency $\omega_{2}$ and the atomic system finally coming back to the initial state $|1\rangle$.

The Hamiltonian for this process can be written as $(\hbar=1)$ :

$$
H=\omega_{1} a^{\dagger} a+\omega_{2} b^{\dagger} b+g\left(a^{3} b^{\dagger}+a^{\dagger 3} b\right)
$$

where $a^{\dagger}(a)$ and $b^{\dagger}(b)$ are the creation (annihilation) operators of the pump field ( $A$-mode) and harmonic field ( $B$-mode), respectively, and $g$ is the coupling constant in the interaction Hamiltonian, which is assumed real, describes the coupling between the two modes of the order of $10^{2}-10^{4}$ per second and also proportional to the nonlinear susceptibility of the medium as well as the complex amplitude of the pump field [20, 21]. In this model $A$ and $B$ are slowly varying operators useful to discuss squeezing defined as $A=a \exp \left(\mathrm{i} \omega_{1} t\right)$ and $B=b \exp \left(\mathrm{i} \omega_{2} t\right)$ with the relation $\omega_{2}=3 \omega_{1}$.

Using the interaction Hamiltonian of Eq. (13) in the coupled Heisenberg equation of motion $(\hbar=1)$ :

$$
\dot{A}=\frac{\partial A}{\partial t}+\mathrm{i}[H, A]
$$

we obtain

$$
\dot{A}=-3 \mathrm{i} g A^{\dagger 2} B \text {. }
$$

Similarly, we have

$$
\dot{B}=-\mathrm{i} g A^{3} \text {. }
$$

The interaction time is taken to be short, of the order of $10^{-10} \mathrm{~s}$ and a nanosecond or picosecond pulse laser can be used as the pump field. For real physical situation in the short-time scale $g t \ll 1\left(g t \approx 10^{-6}\right)$ and the number of photons are very large $\left(|\alpha|^{2} \gg 1\right)$, it is possible to obtain much simpler approximate analytical formulae describing the variances. Expanding $A(t)$ in Taylor's expansion and keep terms up to first-order in $g t$, 


$$
A(t)=A(0)+t \dot{A}(0)+\ldots
$$

In Eq. (17) the operators $A(0) \equiv A$ and $\left.\dot{A}(0) \equiv \frac{\partial A(t)}{\partial t}\right|_{=0}$ without an argument are operators evaluated at $=0$. This convention is followed throughout the paper. Using Eq. (15) in Eq. (17), we obtain

$$
A(t)=A-3 \mathrm{i} g t A^{\dagger 2} B,
$$

then in reversal order

$$
A^{\dagger}(t)=A^{\dagger}+3 \mathrm{i} g t A^{2} B^{\dagger} \text {. }
$$

Similarly,

$$
B(t)=B-\mathrm{i} g t A^{3}
$$

and

$$
B^{\dagger}(t)=B^{\dagger}+\mathrm{i} g t A^{\dagger 3} .
$$

In order to examine the amplitude squeezing of the fundamental mode $A$, as a function of time we define quadrature components

$$
X_{1 A}(t)=\frac{1}{2}\left[A(t)+A^{\dagger}(t)\right]
$$

and

$$
X_{2 A}(t)=\frac{1}{2 \mathrm{i}}\left[A(t)-A^{\dagger}(t)\right] .
$$

Using Eqs. (18) and (19) in Eqs. (22) and (23), we obtain

$$
X_{1 A}(t)=\frac{1}{2}\left[\left(A+A^{\dagger}\right)-3 \operatorname{i} g t\left(A^{\dagger 2} B-A^{2} B^{\dagger}\right)\right]
$$
and

$$
X_{2 A}(t)=\frac{1}{2 \mathrm{i}}\left[\left(A-A^{\dagger}\right)-3 \mathrm{i} g t\left(A^{\dagger 2} B+A^{2} B^{\dagger}\right)\right] .
$$

Now, we assume an initial quantum state as a product of coherent states $|\alpha\rangle$ for the fundamental mode $A$ and $|\beta\rangle$ for the harmonic mode $B$, i.e.

$$
|\psi\rangle=|\alpha\rangle_{A}|\beta\rangle_{B}
$$

Using Eqs. (24) and (26), we obtain

$$
\begin{aligned}
& \left\langle\psi\left|X_{1 A}^{2}(t)\right| \psi\right\rangle=\frac{1}{4}\left[\alpha^{2}+\alpha^{* 2}+2|\alpha|^{2}+1\right. \\
& \quad-6 \mathrm{i} g t\left(|\alpha|^{2} \alpha^{*} \beta+\alpha^{* 3} \beta-\alpha^{3} \beta^{*}-|\alpha|^{2} \alpha \beta^{*}\right. \\
& \left.\left.+\alpha^{*} \beta-\alpha \beta^{*}\right)\right]
\end{aligned}
$$

and

$$
\begin{aligned}
& \left\langle\psi\left|X_{1 A}(t)\right| \psi\right\rangle^{2}=\frac{1}{4}\left[\alpha^{2}+\alpha^{* 2}+2|\alpha|^{2}-6 \mathrm{i} g t\left(|\alpha|^{2} \alpha^{*} \beta\right.\right. \\
& \left.\left.+\alpha^{* 3} \beta-\alpha^{3} \beta^{*}-|\alpha|^{2} \alpha \beta^{*}\right)\right] .
\end{aligned}
$$

Hence the field variance is

$$
\begin{gathered}
{\left[\Delta X_{1 A}(t)\right]^{2}=\left\langle X_{1 A}^{2}(t)\right\rangle-\left\langle X_{1 A}(t)\right\rangle^{2}} \\
\quad=\frac{1}{4}\left[1-6 \mathrm{i} g t\left(\alpha^{*} \beta-\alpha \beta^{*}\right)\right] .
\end{gathered}
$$

From Eqs. (4) and (29) yields

$$
\left[\Delta X_{1 A}(t)\right]^{2}-\frac{1}{4}=3|g t||\alpha \beta| \sin \left(\theta_{2}-\theta_{1}\right)
$$

where $\left\langle N_{A}\right\rangle=\left\langle A^{\dagger} A\right\rangle=|\alpha|^{2}$ and $\left\langle N_{B}\right\rangle=\left\langle B^{\dagger} B\right\rangle=|\beta|^{2}$ are the photon number operators in mode $A$ and $B$, respectively and $\alpha=|\alpha| \exp \left(\mathrm{i} \theta_{1}\right)$ and $\beta=|\beta| \exp \left(\mathrm{i} \theta_{2}\right)$; $\theta_{1}$ and $\theta_{2}$ are the phase angles, $\alpha^{*}, \beta^{*}$ denote the complex conjugate of $\alpha$ and $\beta$, respectively. Equation (30) shows that the squeezing of $X_{1 A}$ will occur whenever $\sin \left(\theta_{2}-\theta_{1}\right)<0$.
Similarly, using Eqs. (25) and (26) for $X_{2 A}$ direction, we have

$$
\left[\Delta X_{2 A}(t)\right]^{2}-\frac{1}{4}=-3|g t||\alpha \beta| \sin \left(\theta_{2}-\theta_{1}\right) .
$$

The right-hand side of Eq. (31) is negative when $\sin \left(\theta_{2}-\right.$ $\left.\theta_{1}\right)>0$, showing the existence of normal squeezing in the fundamental mode up to first order in $g t$ in THG process. From Eqs. (30) and (31) we infer that only one quadrature can be squeezed at a time hence it follows the Heisenberg uncertainty principle.

Further, in order to measure the degree of amplitude squeezing, we define normalized parameter [23] as

$$
Q_{x}=\frac{\left[\Delta X_{2 A}(t)\right]^{2}-1 / 4}{1 / 4}=-12|g t||\alpha \beta| \sin \left(\theta_{2}-\theta_{1}\right) .
$$

Hence the degree of amplitude squeezing in the fundamental mode is found to be dependent on the phase angle of the field amplitude.

We plot a graph of Eq. (32) between the normalized parameter of squeezing $Q_{x}$ and the photon number $|\alpha|$ with different values of $|\beta|$ in Fig. 2.

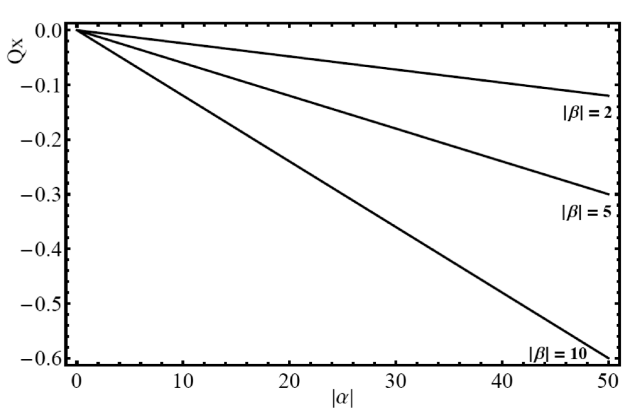

Fig. 2. Dependence of degree of normal amplitude squeezing $Q_{x}$ on $|\alpha|$ and $|\beta|$ in THG process (when $|g t|=10^{-4}$ and $\theta_{1}=0$ and $\theta_{2}=\pi / 2$ ).

Figure 2 shows that the normal squeezing increases linearly with increase of the value of $|\alpha|$. We also observe that when higher the value of $|\beta|$, then squeezing increases and lowers the depth of classicality of the field amplitude. It shows that the degree of normal squeezing directly depends upon the photon number of the fundamental mode as well as on the harmonic mode.

Similarly for studying one of the class of higher-order squeezing like squeezing of amplitude-squared of the fundamental mode as a function of time, we define a real quadrature component for the pump mode:

$$
Y_{1 A}(t)=\frac{1}{2}\left[A^{2}(t)+A^{\dagger 2}(t)\right]
$$

and

$$
Y_{2 A}(t)=\frac{1}{2 \mathrm{i}}\left[A^{2}(t)-A^{\dagger 2}(t)\right] .
$$

Using Eqs. (18), (19) in Eq. (33), we get

$$
Y_{1 A}(t)=\frac{1}{2}\left[A^{2}+A^{\dagger 2}-6 \mathrm{i} g t\left(A^{\dagger 2} A B+A^{\dagger} B\right.\right.
$$




$$
\left.\left.-A^{\dagger} A^{2} B^{\dagger}-A B^{\dagger}\right)\right]
$$

Using Eq. (26) in Eq. (35), we obtain the expectation value as

$$
\begin{aligned}
& \left\langle\psi\left|Y_{1 A}^{2}(t)\right| \psi\right\rangle=\frac{1}{4}\left[\alpha^{4}+\alpha^{* 4}+2|\alpha|^{4}+4|\alpha|^{2}+2\right. \\
& \quad-6 \mathrm{i} g t\left(2|\alpha|^{4} \alpha \beta-2|\alpha|^{4} \alpha^{*} \beta^{*}+6|\alpha|^{2} \alpha \beta\right. \\
& \quad-6|\alpha|^{2} \alpha^{*} \beta^{*}+2|\alpha|^{2} \alpha^{* 3} \beta-2|\alpha|^{2} \alpha^{3} \beta^{*}+4 \alpha^{* 3} \beta \\
& \left.\left.\quad-4 \alpha^{3} \beta^{*}+4 \alpha \beta-4 \alpha^{*} \beta^{*}\right)\right]
\end{aligned}
$$

and

$$
\begin{aligned}
& \left\langle\psi\left|Y_{1 A}(t)\right| \psi\right\rangle^{2}=\frac{1}{4}\left[\alpha^{4}+\alpha^{* 4}+2|\alpha|^{4}-6 \mathrm{i} g t\left(2|\alpha|^{4} \alpha \beta\right.\right. \\
& -2|\alpha|^{4} \alpha^{*} \beta^{*}+2|\alpha|^{2} \alpha \beta-2|\alpha|^{2} \alpha^{*} \beta^{*}+2|\alpha|^{2} \alpha^{* 3} \beta \\
& \left.\left.-2|\alpha|^{2} \alpha^{3} \beta^{*}+2 \alpha^{* 3} \beta-2 \alpha^{3} \beta^{*}\right)\right],
\end{aligned}
$$

and the expectation value of the time dependent mean photon number is

$$
\left\langle N_{A}(t)\right\rangle=|\alpha|^{2}-3 \mathrm{i} g t\left(\alpha^{* 3} \beta-\alpha^{3} \beta^{*}\right) .
$$

Using Eqs. (36) and (37) in Eq. (7), we get

$$
\begin{aligned}
& {\left[\Delta Y_{1 A}(t)\right]^{2}-\left\langle N_{A}(t)+1 / 2\right\rangle=12|g t||\alpha \beta| \sin \left(\theta_{1}+\theta_{2}\right)} \\
& \quad \times\left[\left(|\alpha|^{2}+1\right)\right] .
\end{aligned}
$$

Similarly, we get for $Y_{2 A}$ quadrature as

$$
\begin{aligned}
& {\left[\Delta Y_{2 A}(t)\right]^{2}-\left\langle N_{A}(t)+1 / 2\right\rangle} \\
& \quad=-12|g t||\alpha \beta| \sin \left(\theta_{1}+\theta_{2}\right)\left[\left(|\alpha|^{2}+1\right)\right] .
\end{aligned}
$$

The right-hand side of Eqs. (39) and (40) are showing the existence of squeezing in amplitude-squared in the fundamental mode in THG process when $\sin \left(\theta_{1}+\theta_{2}\right)<0$ and $\sin \left(\theta_{1}+\theta_{2}\right)>0$, respectively. The multiplication factor $\left(|\alpha|^{2}+1\right)$ is the nonlinear effect due to strong pump field interaction.

Now, we define the normalized parameter [23] for amplitude-squared squeezing as,

$$
\begin{aligned}
& Q_{y}=\frac{\left[\Delta Y_{2 A}(t)\right]^{2}-\left\langle\left(N_{A}(t)+1 / 2\right)\right\rangle}{\left\langle\left(N_{A}(t)+1 / 2\right)\right\rangle} \\
& =\frac{-12|g t||\alpha \beta| \sin \left(\theta_{1}+\theta_{2}\right)\left[\left(|\alpha|^{2}+1\right)\right]}{\left(|\alpha|^{2}+1 / 2\right)+6|g t|\left|\alpha^{3} \beta\right| \sin \left(\theta_{2}-3 \theta_{1}\right)} .
\end{aligned}
$$

Hence the degree of amplitude-squared (second-order) squeezing in the fundamental mode directly depends upon the phase angle of the field amplitude up to the first-order in $g t$. The variation of $Q_{y}$ with $|\alpha|^{2}$ is shown in Fig. 3.

In Fig. 3 the steady fall of the curve shows that the squeezing increases nonlinearly with $|\alpha|^{2}$, which is directly dependent upon the number of photons. This confirms that the squeezed states are associated with a large number of photons. We also find that when higher the value of $|\beta|$, then the squeezing increases and lowers the depth of classicality of the field amplitude. It confirms that the degree of squeezing directly depends upon the photon number of the fundamental field as well as on the harmonic field.

A comparison between Figs. 2 and 3 show greater noise reduction in higher-order (amplitude-squared) than the

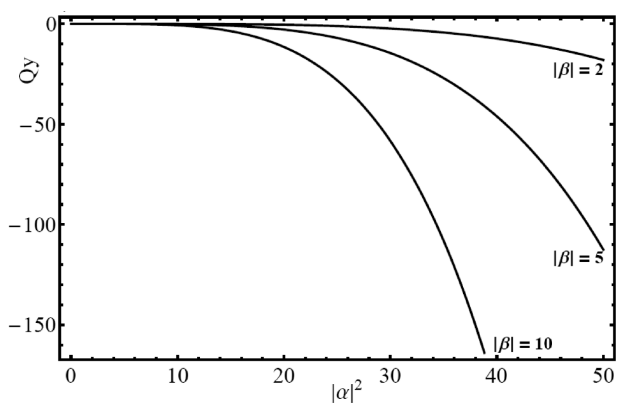

Fig. 3. Dependence of degree of higher-order (amplitude-squared) squeezing $Q_{y}$ on $|\alpha|^{2}$ and $|\beta|$ in THG process (when $|g t|=10^{-4}$ and $\theta_{1}=0$ and $\left.\theta_{2}=\pi / 2\right)$.

first-order (normal squeezing), having the same number of photons.

Now, using Eq. (38), we obtain expectation values of mean photon number as

$$
\left\{\begin{array}{l}
\left\langle N_{A}^{2}(t)\right\rangle=|\alpha|^{4}+|\alpha|^{2}-3 \mathrm{i} g t\left(2|\alpha|^{2} \alpha^{* 3} \beta\right. \\
\left.\quad-2|\alpha|^{2} \alpha^{3} \beta^{*}+3 \alpha^{* 3} \beta-3 \alpha^{3} \beta^{*}\right), \\
\left\langle N_{A}(t)\right\rangle^{2}=|\alpha|^{4}-3 \mathrm{i} g t\left(2|\alpha|^{2} \alpha^{* 3} \beta-2|\alpha|^{2} \alpha^{3} \beta^{*}\right) .
\end{array}\right.
$$

Hence the fluctuation of time dependent mean photon number is

$$
\begin{aligned}
& {\left[\Delta N_{A}(t)\right]^{2}=\left\langle N_{A}^{2}(t)\right\rangle-\left\langle N_{A}(t)\right\rangle^{2}} \\
& \quad=|\alpha|^{2}+18 g t\left|\alpha^{3} \beta\right| \sin \left(\theta_{2}-3 \theta_{1}\right)
\end{aligned}
$$

and the photon statistics of pump mode in THG is found to be sub-Poissonian, as

$$
\left[\Delta N_{A}(t)\right]^{2}-\left\langle N_{A}(t)\right\rangle=12|g t|\left|\alpha^{3} \beta\right| \sin \left(\theta_{2}-3 \theta_{1}\right) .
$$

The right-hand side of Eq. (44) is always negative when $\sin \left(-3 \theta_{1}+\theta_{2}\right)<0$, showing the existence of sub-Poissonian light in the fundamental mode up to the first order in $g t$ under short-time approximation in THG process. It indicates that the occurrence of sub-Poissonian light in the fundamental mode directly depends upon the phase angle of the field amplitude. The present results show that for particular phase values of $\theta_{1}=0$ and $\theta_{2}=3 \pi / 2$, squeezing and sub-Poissonian photon statistics in the fundamental mode are always negative. Thus we infer that sub-Poissonian effects of light appear simultaneously with the amplitude squeezing to the case of first order approximation in coupling constant.

\section{Squeezing effects of light in the harmonic mode}

To examine squeezing in the harmonic mode $B$ as a function of time, we define the quadrature operators

$$
X_{1 B}(t)=\frac{1}{2}\left[B(t)+B^{\dagger}(t)\right]
$$

and

$$
X_{2 B}(t)=\frac{1}{2 \mathrm{i}}\left[B(t)-B^{\dagger}(t)\right] .
$$

Using Eqs. (20) and (21) in Eqs. (45) and (46), we find 
and

$$
X_{1 B}(t)=\frac{1}{2}\left[\left(B+B^{\dagger}\right)-\mathrm{i} g t\left(A^{3}-A^{\dagger^{3}}\right)\right]
$$

$$
X_{2 B}(t)=\frac{1}{2 \mathrm{i}}\left[\left(B-B^{\dagger}\right)-\mathrm{i} g t\left(A^{3}+A^{\dagger^{3}}\right)\right] .
$$

Using Eq. (26) in Eq. (47), we obtain as

$$
\begin{array}{r}
\left\langle\psi\left|X_{1 B}^{2}(t)\right| \psi\right\rangle=\frac{1}{4}\left[\beta^{2}+\beta^{* 2}+2|\beta|^{2}+1\right. \\
\left.-2 \mathrm{i} g t\left(\alpha^{3} \beta+\alpha^{3} \beta^{*}-\alpha^{* 3} \beta-\alpha^{* 3} \beta^{*}\right)\right]
\end{array}
$$

and

$$
\begin{aligned}
& \left\langle\psi\left|X_{1 B}(t)\right| \psi\right\rangle^{2}=\frac{1}{4}\left[\beta^{2}+\beta^{* 2}+2|\beta|^{2}\right. \\
& \left.-2 \mathrm{i} g t\left(\alpha^{3} \beta+\alpha^{3} \beta^{*}-\alpha^{* 3} \beta-\alpha^{* 3} \beta^{*}\right)\right] .
\end{aligned}
$$

Hence

$$
\left[\Delta X_{1 B}(t)\right]^{2}=\left\langle X_{1 B}^{2}(t)\right\rangle-\left\langle X_{1 B}(t)\right\rangle^{2}=\frac{1}{4} .
$$

Similarly from Eq. (48), we have

$$
\left[\Delta X_{2 B}(t)\right]^{2}=\frac{1}{4} \text {. }
$$

From Eqs. (51) and (52), we can see that the $B$-mode is initially in a coherent state, i.e.

$$
\left[\Delta X_{1 B}\right]^{2}=\left[\Delta X_{2 B}\right]^{2}=\frac{1}{4} \text {. }
$$

Hence there is no possibility to produce squeezed light in the harmonic mode up to the first-order approximation in $g t$.

It is now interesting to study squeezing in the $B$-mode (harmonic) up to second order approximation in $g t$ in the fundamental mode.

From Eq. (16) we obtain as

$$
\ddot{B}=-g^{2}\left(9 N_{A}^{2}+9 N_{A}+6\right) B .
$$

Using Eq. (17) and the corresponding results in the harmonic mode up to second order in $g t$, is as follows:

$$
B(t)=B(0)-\mathrm{i} g t A^{3}-\frac{1}{2}|g t|^{2}\left(9 N_{A}^{2}+9 N_{A}+6\right) B
$$

and

$$
B^{\dagger}(t)=B^{\dagger}(0)+\mathrm{i} g t A^{\dagger 3}-\frac{1}{2}|g t|^{2}\left(9 N_{A}^{2}+9 N_{A}+6\right) B^{\dagger} .
$$

Using Eqs. (55) and (56) in Eqs. (45) and (46), we get

$$
\begin{gathered}
X_{1 B}(t)=X_{1 B}+g t Z_{2 A}-\frac{1}{2}|g t|^{2} \\
\quad \times\left(9 N_{A}^{2}+9 N_{A}+6\right) X_{1 B}
\end{gathered}
$$

and

$$
\begin{aligned}
& X_{2 B}(t)=X_{2 B}-g t Z_{1 A}-\frac{1}{2}|g t|^{2} \\
& \quad \times\left(9 N_{A}^{2}+9 N_{A}+6\right) X_{2 B} .
\end{aligned}
$$

For an uncorrelated modes at $=0$, we get

$$
\begin{gathered}
{\left[\Delta X_{1 B}(t)\right]^{2}=\left(\Delta X_{1 B}\right)^{2}+|g t|^{2}\left[\left(\Delta Z_{2 A}\right)^{2}\right.} \\
\left.-\left\langle\left(9 N_{A}^{2}+9 N_{A}+6\right)\right\rangle\left(\Delta X_{1 B}\right)^{2}\right]
\end{gathered}
$$

and

$$
\begin{gathered}
{\left[\Delta X_{2 B}(t)\right]^{2}=\left(\Delta X_{2 B}\right)^{2}+|g t|^{2}\left[\left(\Delta Z_{1 A}\right)^{2}\right.} \\
\left.-\left\langle\left(9 N_{A}^{2}+9 N_{A}+6\right)\right\rangle\left(\Delta X_{2 B}\right)^{2}\right] .
\end{gathered}
$$

$$
\begin{aligned}
& {\left[\Delta X_{1 B}(t)\right]^{2}-\frac{1}{4}} \\
& \quad=|g|^{2} t^{2}\left[\left(\Delta Z_{2 A}\right)^{2}-\frac{1}{4}\left\langle\left(9 N_{A}^{2}+9 N_{A}+6\right)\right\rangle\right]
\end{aligned}
$$

and

$$
\begin{aligned}
& {\left[\Delta X_{2 B}(t)\right]^{2}-\frac{1}{4}} \\
& \quad=|g|^{2} t^{2}\left[\left(\Delta Z_{1 A}\right)^{2}-\frac{1}{4}\left\langle\left(9 N_{A}^{2}+9 N_{A}+6\right)\right\rangle\right] .
\end{aligned}
$$

Equations (61) and (62) show that $X_{1 B}$ in the harmonic mode is squeezed if $Z_{2 A}$ is squeezed and $X_{2 B}$ is squeezed if $Z_{1 A}$ is squeezed. In other words, the $B$-mode is squeezed in the $X_{1 B}$ direction if the $A$-mode is amplitude-cubed squeezed in the $Z_{2 A}$ direction and the $B$-mode is squeezed in the $X_{2 B}$ direction if the $A$-mode is amplitude-cubed squeezed in the $Z_{1 A}$ direction. That is, if a fundamental mode with amplitude-cubed squeezing propagates through a nonlinear medium a squeezed harmonic mode is generated.

Now, comparing the results of Eq. (62) with earlier published work [22], then we have as (we consider here only the $Z_{1 A}(t)$ quadrature)

$$
\begin{gathered}
{\left[\Delta X_{2 B}(t)\right]^{2}-\frac{1}{4}=-\frac{9}{4}|g t|^{4}\left[6\left(|\alpha|^{8}+2|\alpha|^{6}\right) \cos 6 \theta\right.} \\
\left.\quad+9|\alpha|^{8}+69|\alpha|^{6}+138|\alpha|^{4}+60|\alpha|^{2}+4\right] .
\end{gathered}
$$

The right-hand side of Eq. (63) is always negative, showing the existence of normal squeezing in the harmonic mode in $X_{2 B}$ quadrature which is $|g t|^{2}$ times of third-order squeezing in $Z_{1 A}(t)$ quadrature [22]. This also establishes the fact that the occurrence of squeezing in the harmonic mode is only due to the presence of the fundamental mode. An analysis of Eq. (63) shows that if $g^{2} t^{2}<1$ squeezing is greater in harmonic mode compared to fundamental mode.

To study optimum squeezing in harmonic mode, we denote the right hand side of Eq. (63) by $S_{X}$. Taking $|g t|^{2}=10^{-4}$ and $\theta=0$ as it gives maximum squeezing. The variation of $S_{X}$ with $|\alpha|^{2}$ is shown in Fig. 4.

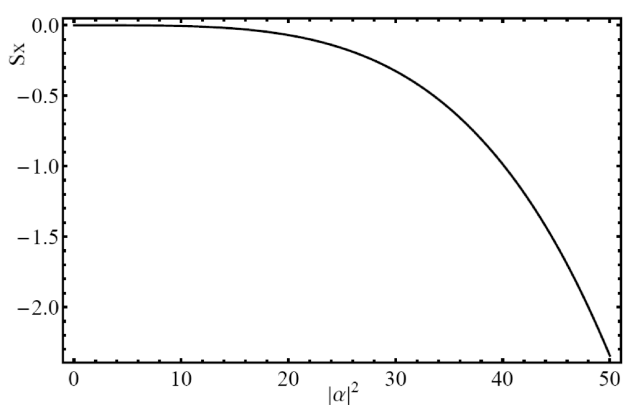

Fig. 4. Dependence of harmonic squeezing $S_{x}$ with $|\alpha|^{2}$ in THG process (when $|g t|^{2}=10^{-4}$ and $\theta=0$ ).

Figure 4 shows that the squeezing increases nonlinearly with $|\alpha|^{2}$, which is directly dependent upon the number of photons. This again confirms that squeezed states are associated with a large number of photons. Moreover, the 
curve establishes the relationship between the harmonic and the fundamental mode.

\section{Conclusions}

The important findings of this paper are as follows. In this paper the squeezing and sub-Poissonian effects of light of the fundamental mode in third harmonic generation are investigated up to the first order Hamiltonian interaction in $g t$. It is shown that amplitude and amplitude-squared squeezing as well as sub-Poissonian photon statistics of light in the fundamental mode is directly dependent upon the selective phase values of the field amplitude in both the quadratures. A comparison between first- and second-order squeezing shows greater squeezing in second-order having the same number of photons. It is inferred that higher-order squeezing (amplitude-squared) makes it possible to achieve significantly larger noise reduction than ordinary (normal) squeezing. The occurrence of multiplication factor $\left(|\alpha|^{2}+1\right)$ in the second-order squeezing is the nonlinear effect due to strong pump field interaction, which shows that squeezing is found to be maximum in amplitude-squared followed by amplitude-squeezing. It is also found that the degree of squeezing and occurrence of sub-Poissonian light directly depends upon the photon number of the fundamental field as well as on the harmonic field. In all cases degree of squeezing increases and lowers the depth of classicality of field amplitude with photon number. It is shown that for the particular phase values the amplitude squeezing and sub-Poissonian photon statistics of light appear simultaneously.

In the harmonic mode, we have found that there is no possibility to produce squeezed laser light in the harmonic mode to the case of first-order Hamiltonian interaction in coupling constant. Further, we have extended the case up to second-order interaction in $g t$ and found that the normal squeezing in the harmonic mode directly depends upon the amplitude-cubed squeezing of the initial pump field. This gives a method of converting higher-order (amplitude-cubed) squeezing of the fundamental mode into normal squeezing of the harmonic mode and vice versa. It is observed that first-order harmonic squeezing in $X_{2 B}$ quadrature is $|g t|^{2}$ times of the third-order fundamental squeezing in $Z_{1 A}$ quadrature. Moreover, we have found that if $g^{2} t^{2}<1$, squeezing is greater in harmonic mode compared to fundamental mode.

As a result, this family of squeezing and sub-Poissonian photon statistics effects in relation to selective phase values of the field amplitude of the fundamental as well as of the harmonic mode is useful to obtain the desired degree of amplitude squeezing through different higher-order nonlinear optical processes. Hence, these results may pave the way for obtaining greater noise reduction in optical systems and can be useful in high quality telecommunication.

\section{Acknowledgments}

We would like to thank the referee for his valuable suggestions.

\section{References}

[1] D.F. Walls, Nature 306, 141 (1983); R. Loudon, P.L. Knight, J. Mod. Opt. 34, 709 (1987); M.C. Teich, B.E.A. Saleh, Quant. Opt. 1, 153 (1989).

[2] J. Perina, Quantum Statistics of Linear and Nonlinear Optical Phenomena, Kluwer, Dordrecht 1999, Ch. 9 and 10; L. Mandel, Phys. Scr. T 12, 34 (1986); V.V. Dodonov, J. Opt. B: Quant. Semiclass. Opt. 4, R1 (2002).

[3] B.E.A. Saleh, M.C. Teich, Phys. Rev. Lett. 58, 2656 (1987).

[4] Special issues devoted to squeezed states: K. Wódkiewicz, J. Mod. Opt. 34, 941 (1987); H.J. Kimble, D.F. Walls, J. Opt. Soc. Am. B 4, 1450 (1987).

[5] H.P. Yuen, J.H. Shapiro, IEEE Trans. Inf. Theory 24, 657 (1978).

[6] C.H. Bennett, G. Brassard, N.D. Mermin, Phys. Rev. Lett. 68, 557 (1992); J. Kempe, Phys. Rev. A 60, 910 (1999).

[7] J. Peřina, V. Peřinová, J. Kodousek, Opt. Commun. 49, 210 (1984).

[8] S. Kielich, R. Tanas, R. Zawodny, J. Opt. Soc. Am. B 4, 1627 (1987)

[9] J. Peřina, V. Peřinova, C. Sibilia, M. Bertolotti, Opt. Commun. 49, 285 (1984).

[10] M.S.K. Razmi, J.H. Eberly, Opt. Commun. 76, 265 (1990).

[11] D.K. Giri, P.S. Gupta, J. Opt. B: Quant. Semiclass. Opt. 6, 91 (2004); Opt. Commun. 221, 135 (2003).

[12] J. Perina, J. Krepelka, J. Mod. Opt. 38, 2137 (1991).

[13] A. Kumar, P.S. Gupta, Quant. Semiclass. Opt. 7, 835 (1995).

[14] A. Kumar, P.S. Gupta, Quant. Semiclass. Opt. 8, 1053 (1996)

[15] C.K. Hong, L. Mandel, Phys. Rev. Lett. 54, 323 (1985); Phys. Rev. A 32, 974 (1985).

[16] M. Hillery, Opt. Commun. 62, 135 (1987); Phys. Rev. A 36, 3796 (1987); Phys. Rev. A 45, 4944 (1992).

[17] L. Mandel, Opt. Commun. 42, 437 (1982).

[18] Y. Kim, T.H. Yoon, Opt. Commun. 212, 107 (2002).

[19] H. Prakash, D.K. Mishra, J. Phys. B, At. Mol. Opt. Phys. 39, 2291 (2006); Opt. Commun. 283, 3284 (2010).

[20] R. Tanas, Phys. Lett. A 141, 217 (1989).

[21] R. Tanas, A. Miranowicz, S. Kielich, Phys. Rev. A 43, 4014 (1991).

[22] Y.-B. Zhan, Phys. Lett. A 160, 498 (1991).

[23] L. Mandel, Opt. Lett. 4, 205 (1979). 